\title{
Passive repetitive stretching increases skeletal muscle mass and myofiber cross-sectional area in senescence-accelerated model mouse
}

\section{Yumin Wang}

Department of rehabilitation and physical medicine, Hokkaido University Graduate School of Medicine Satoshi Ikeda ( $\square$ ikedas@med.hokudai.ac.jp)

Department of rehabilitation and physical medicine, Hokkaido University Hospital.

\section{Katsunori Ikoma}

Department of rehabilitation and physical medicine, Hokkaido University Hospital.

\section{Research Article}

Keywords: Akt, p70S6K, 4E-BP1, Myf5, myogenin, MuRF1, injuries, physical disability, frailty

Posted Date: February 23rd, 2021

DOl: https://doi.org/10.21203/rs.3.rs-235691/v1

License: (1) This work is licensed under a Creative Commons Attribution 4.0 International License.

Read Full License

Version of Record: A version of this preprint was published at Scientific Reports on July 27th, 2021. See the published version at https://doi.org/10.1038/s41598-021-94709-0. 


\section{Abstract}

Mechanical stimulation has benefits for muscle mass and function. Passive stretching is widely performed in clinical rehabilitation medicine. However, the hypertrophic effects of passive repetitive stretching on senescent skeletal muscles against muscle atrophy remain unknown. We used senescenceaccelerated model SAM-P8 mice. The gastrocnemius muscle was passively repetitive stretched by manual ankle dorsiflexion for $15 \mathrm{~min}, 5$ days a week for 2 weeks under deep anesthesia. We examined the effects of passive stretching on muscle mass, myofiber cross-sectional area, muscle fiber type and composition, satellite cell content, mRNA expression of the signaling pathways involved in muscle protein synthesis, muscle-specific ubiquitin ligases, and myogenic regulatory factors. The gastrocnemius muscle weight of the stretched side increased compared with that of the unstretched side. In addition to the increase in muscle mass, muscle fiber cross-sectional area of the stretched side was greater than that of the unstretched side. Passive repetitive stretching significantly increased the mRNA expression level of Akt, p70S6K, 4E-BP1, Myf5, myogenin, MuRF1. Passive repetitive stretching promoted skeletal muscle mass and myofiber cross-sectional area in SAM-P8 mice. These hypertrophic observations are attributable to the stretch-activated signaling pathways involved in protein turnover. These findings are applicable to clinical muscle strengthening and sarcopenia prevention.

\section{Introduction}

Age-associated loss of muscle mass and function, known as sarcopenia, is characterized by a progressive decline in muscle fiber number and size, a shift in fiber-type composition, and modification of adverse metabolic parameters ${ }^{1,2}$. With the increasing risk of multiple outcomes such as fall-related injuries, physical disability, and frailty, sarcopenia has become a major threat to independence and quality of life in older individuals worldwide ${ }^{1,2}$. Despite the growing recognition of etiologies and clinical importance, sarcopenia is poorly managed by routine treatment. It is imperative to identify effective countermeasures that induce senescent muscle hypertrophy and combat progression of sarcopenia.

Passive stretching, a type of mechanical stimulus, is widely used in rehabilitation medicine to prevent muscle shortening, maintain the range of joints, and muscle flexibility ${ }^{3,4}$. Some studies indicate that passive stretching may induce muscle hypertrophy by triggering mechanisms such as satellite cells and myogenic growth factors, mechanically activated ion channels, anabolic signaling, and protein synthesis $^{5-9}$. It is performed with minimal risk of injury at relatively light intensity as compared with resistant exercise and is used to prevent muscle disuse or enhance recovery from exposure to prolonged inactivity ${ }^{8.10}$. However, aging processes alter mechano-transduction, which is the ability for skeletal muscle cells perceive and respond to mechanical inputs, indicating a perturbed load-induced plasticity for aged muscle hypertrophy ${ }^{11}$. As such, divergences in the stretching protocols applied also affect muscular hypertrophic adaptations. According to previous reports, intermittent stretching performed for 1 week in aged rats resulted in unchanged muscle mass, whereas continuous stretching performed using a splint for 4 weeks provoked an increase in the weight of aged soleus and plantaris muscles ${ }^{12,13}$. Stretching by 
immobilization induces articular contracture and is not commonly used in routine rehabilitation practice. By contrast, no studies have demonstrated the effect of daily repetitive stretching for short durations on senescent skeletal muscles. Prior reports highlighted the advantages of daily repetitive stretching in regulating the myogenic regulatory factors and suppressing denervation-induced atrophy through the Akt/mTOR signaling cascade ${ }^{6,7,9}$. These findings suggest a potential hypertrophic effect of repetitive stretching, which prevents sarcopenic atrophy in senescent skeletal muscles.

Disrupted protein homeostasis is well established as an initial process that regulates age-related muscle mass reduction ${ }^{14}$. Skeletal muscle atrophy occurs when protein degradation rates transcend rates of protein synthesis ${ }^{15}$. Hence, strategies that enhance protein synthesis may prevent sarcopenic progression. IGF1/Akt/mTOR signaling primarily controls muscle protein synthesis ${ }^{14,15}$. Akt stimulates protein synthesis by activating mTOR and inhibits protein degradation through FoxO-mediated ubiquitinligases including Muscle Atrophy F-box (MAFbx)/atrogin-1, and Muscle Ring Finger 1 (MuRF1) downregulation ${ }^{14,15}$. Myostatin, a member of the TGF- $\beta$ superfamily, has been extensively studied as another potential mediator of sarcopenia owing to its potent negative effects on cell growth and intracellular catabolic and anabolic signaling pathways ${ }^{16}$.

Skeletal muscle hypertrophy can be induced by the activation of muscle satellite cells. Once satellite cells are activated, myogenic regulatory factors (MRFs), including MyoD, Myf5, and myogenin, play a pivotal role in skeletal muscle formation and development ${ }^{17}$. Markers of early myogenesis, MyoD and Myf5, contribute to determination, while the marker of late myogenesis, myogenin, plays a downstream role in muscle cell differentiation to form muscle fibers ${ }^{17}$. However, accumulating evidence suggests that satellite cell numbers and responsiveness are altered during aging, which may be associated with sarcopenia ${ }^{18}$. Although satellite cells are indispensable for muscle regeneration, whether they play a key role in the maintenance of skeletal muscle mass and myofiber size throughout life is still controversial.

Thus, the aim of this study was to investigate the impact of passive repetitive stretching on senescent skeletal muscles by using senescence-accelerated mouse prone-8 (SAM-P8), which exhibits characteristics of accelerated muscle aging, and is reported to be valid for muscular aging research ${ }^{14}$. We hypothesized that passive repetitive stretching induces muscle hypertrophy in SAM-P8 mice. We investigated the effects of passive repetitive stretching in terms of muscle mass, fiber size, muscle fibertype composition, satellite cell content, MRFs, and regulatory factors related to muscle protein synthesis.

\section{Results}

\section{Muscle mass and muscle fiber cross-sectional area}

There was no significant difference in body weight between the pre -and post-stretching animals (Figure $2 A)$. The gastrocnemius muscle weight of the stretched side increased by $10.7 \%$ as compared with that of the unstretched side $(P=0.028$; Figure $2 B)$. In addition to the increase in muscle mass, histological analysis using H\&E staining showed that the mean gastrocnemius muscle fiber cross-sectional area of 
the stretched side was $19.9 \%$ larger than that of the unstretched side $(P=0.012$; Figure $2 C, 2 D)$. The frequency distribution of the muscle fiber area further revealed that the stretched gastrocnemius muscle had a shift toward larger myofibers as compared with the unstretched side (Figure 2E).

\section{Muscle fiber type and satellite cells content}

No clear differences in the distribution of fiber types were observed when the fiber-type composition was examined with $\mathrm{IHC}$ staining (Figure $3 \mathrm{~A}$ ). Type 2A fibers were the most common in the gastrocnemius muscle, with lower proportions of type $2 \mathrm{~B}$ and type 1 fibers (Figure $3 \mathrm{~B}$ ). This suggests that fiber-type switching is not regulated by passive repetitive stretching for a short duration within 2 weeks. Pax7 expression has been recognized as a marker of satellite cells. Pax7+ cells were rare, and there was no significant difference in the number of Pax7+ cells between the stretched and unstretched sides (Figure 4).

\section{mRNA expression of Akt, p70S6K, 4E-BP1, MAFbx and MuRF1, MRFs and myostatin.}

The messenger RNA expression levels of Akt/mTOR pathway signal molecules, Akt, p70S6K, and 4E-BP1 are affected by passive repetitive stretching (Figure 5). The mRNA expression of Akt increased by 2.5 -fold $(P=0.04995$; Figure 5A) on the stretched side as compared with the unstretched side. 4E-BP1 and p70S6K are two downstream targets of mTORC1. The mRNA expression of p70S6K increased by 4.9-fold $(P=0.012$; Figure 5B) and 4E-BP1 increased by 5.7-fold ( $P=0.012$; Figure $5 \mathrm{C})$ in the stretched side as compared with the unstretched side. We then measured the expression of major muscle-specific E3 ubiquitin ligases, including MAFbx and MuRF1. The MAFbx mRNA expression was not altered (Figure 5D), whereas the MuRF1 mRNA expression increased by 2.1-fold ( $P=0.04995$; Figure 5E) on the stretched side as compared with the unstretched side.

The expressions of MRFs, including MyoD, Myf5, and myogenin mRNA, are shown in Figure 6. The MyoD mRNA expression remained unchanged (Figure 6A). Myf5 increased by 3.8-fold ( $P=0.017$; Figure 6B) and myogenin increased by 2.7 -fold $(P=0.012$; Figure $6 \mathrm{C})$ on the stretched side as compared with the unstretched side. Myostatin mRNA expression did not differ between the stretched and unstretched sides (Figure 6D).

\section{Discussion}

The findings of this study indicate that passive repetitive stretching for 2 weeks induced an increase in skeletal muscle mass and fiber size and promoted a positive protein turnover in the skeletal muscle via Akt-mTOR signaling pathways, accompanied by an elevated expression level of MuRF1, which is involved in protein degradation, in the senescent skeletal muscles of SAM-P8 mice. To the best of our knowledge, this is the first study to demonstrate muscular hypertrophic adaptation to passive repetitive stretching in aged mice in vivo. 
The theoretical basis for stretch-induced skeletal muscle hypertrophy dates back to in vitro studies. Goldberg et al. found that repetitive stretching applied to cultured skeletal muscle cells provided mechanical stimuli and triggered cellular biomarkers essential for muscle growth ${ }^{20}$. Indeed, robust hypertrophy was observed after progressive stretch overload of the wing muscles in birds ${ }^{21}$. Stretch parameters such as frequency and duration have been identified as important factors that potentially affect the skeletal muscle adaptive process, as the gene expressions involved in muscle growth and atrophy are responsive to the number of stretch sessions ${ }^{22,23}$. Sarcopenia is an age-related reduction in both muscle mass and quality ${ }^{1.2}$. Although prior studies have demonstrated that senescent muscles preserved the capacity to undergo hypertrophy, the ability to perceive and respond to mechanical inputs and translate them into biochemical signals, which is called "mechano-transduction," was reportedly blunted during aging ${ }^{11}$. The impact of aging on the cellular mechano-transduction process is rooted in multiple factors such as modifications in cell cytoskeleton structures, alterations in mechanosensitive signaling, and the extracellular matrix environment ${ }^{11}$. Zotz et al. found that 1 week of intermittent stretching in aged rats resulted in an unchanged muscle mass, accompanied by reduced fiber size ${ }^{12}$. Hotta et al. reported an increase in soleus and plantaris muscle weights after 4 weeks of continuous stretching without further histological analysis of muscle characteristics ${ }^{13}$. As the rats were awake during continuous muscle stretching, it became difficult to isolate the effects of stretching from isometric contractile activity. In another study, contractions were eliminated by animal anesthesia during stretching, while muscle weight and fiber area remained unaltered ${ }^{24}$. In our study, we found that clinically feasible protocols of passive repetitive stretching markedly increased the gastrocnemius muscle mass and myofiber size. This has been proven to be a practical therapeutic approach to induce muscle hypertrophy in senescent muscles. Sarcopenia predominantly affects type 2 muscle fibers, whereas type 1 fibers are less affected ${ }^{2}$. Guo et al. demonstrated that type 2 muscle fibers, which made up the largest proportion of the gastrocnemius muscle, peaked at 8 months, followed by a gradual decline in SAM-P8 mice ${ }^{19}$. However, it appears that muscle fiber-type composition seemed not to be susceptible to regulation within 2 weeks of stretching. Fiber-type plasticity within skeletal muscle is regulated by a sophisticated signaling network with two major pathways, calcineurin signaling and AMP-activated protein kinase (AMPK) signaling with a major mediator, PGC- $1 \mathrm{a}^{25}$. Our observations suggest that the hypertrophic effect of passive repetitive stretching occurred without modifying the fiber-type composition within a short duration of 2 weeks.

The regulation of muscle mass and fiber size substantially reflects changes in protein homeostasis, i.e. the balance between protein synthesis and degradation ${ }^{15}$. Therefore, to decipher the mechanism of action behind the hypertrophic effect of passive repetitive stretching, we first verified whether the Akt/mTOR pathway, which predominantly controls muscle protein synthesis, was involved in muscular adaptation. Akt is an upstream regulator of $\mathrm{mTOR}$, and it is widely recognized that signaling by mTOR is a core module of the pathway through which mechanical stimuli regulate protein synthesis and muscle growth $^{26}$. The regulation is primarily mediated by two downstream targets of the mTOR complex 1 (mTORC1), translational suppressor 4E-BP1 and ribosomal protein $\mathrm{p} 70 \mathrm{~S} 6 \mathrm{~K}^{27}$. It has been reported that 
skeletal muscle stretching activates these signaling molecules, including Akt, p70S6K, and 4E-BP1 in vitro $^{28,29}$. Enhanced Akt, p70S6K, and 4E-BP1 phosphorylation were observed in denervated mice soleus muscles when subjected to repetitive stretching in vivo ${ }^{9}$. Several studies have demonstrated that the responsiveness of Akt/mTOR signaling is diminished in overload-induced muscle growth during aging, suggesting limited plasticity for aged muscle hypertrophy ${ }^{30}$. This study provides evidence that in vivo repetitive stretching strongly increases the expression of Akt, p70S6K, and 4E-BP1 in senescent skeletal muscles at the transcriptional level. The stretch-activated Akt/mTOR signaling pathway involved in skeletal muscle protein synthesis is intact in aged mice.

Moreover, Akt normally blocks the upregulation of several ubiquitin-proteasome genes related to protein degradation in skeletal muscles by negatively regulating FoxO transcription factors ${ }^{15}$. In skeletal muscles, the major muscle-specific ubiquitin ligases include MAFbx/atrogin-1 and MuRF1, which are associated with myonuclear apoptosis and muscle atrophy ${ }^{15}$. However, an expected suppressive effect on MuRF1 and MAFbx was not observed in our study. Peviani et al. found an increase in MAFbx expression in the soleus muscles of rats when daily bouts of stretch were performed ${ }^{23}$. Russo et al. reported that stretching could reduce the accumulation of MAFbx and MuRF1 in a denervated rat skeletal muscle ${ }^{22}$. Furthermore, Soares et al. showed time-course alternations of MAFbx and MuRF1 that decreased drastically after 24-h stretching and then partially recovered after 48- and 96-h stretching in immobilized muscles ${ }^{31}$. These divergent findings suggest that proteasome activity is potentially influenced by stretching protocols or responds differently under physiological and pathological conditions. The alternation of MAFbx and MuRF1 expression in aged skeletal muscle has been reported to be inconsistent. Several studies found that the expression levels of MAFbx and MuRF1 increased in skeletal muscles with aging, which may contribute to sarcopenia ${ }^{32,33}$. However, unaltered and even decreased expression levels of MAFbx and MuRF1 have been shown in other studies ${ }^{34,35}$. In our study, MuRF1 mRNA expression was elevated, indicating the involvement of the cellular degradation pathway in aged skeletal muscle adaptation to passive stretching. In combination with the greater muscle mass and increased muscle fiber size, this may suggest that stretch-induced hypertrophy of senescent muscles may result from relatively enhanced overall rates of protein synthesis that possess a superior position in protein homeostasis during the experimental period. Likewise, differential expression patterns of myostatin in stretching have been observed in previous reports $22,23,36$. Myostatin negatively regulates skeletal muscle growth, primarily by acting via activin type II receptors (ActRII), resulting in the activation of Smad signaling ${ }^{14,15}$. Smad signaling suppresses Akt signaling and its downstream effectors such as mTOR and FoxO to regulate muscle growth ${ }^{14,15}$. Alterations in myostatin expression and signaling activity in the context of aging are not completely understood. We could speculate that passive stretching is a potential intervention to counter, at least in part, sarcopenia via myostatin inhibition. However, myostatin expression was not affected by stretching in our study. A further time-course study may help to define the myostatin expression alternation in response to passive repetitive stretching of senescent skeletal muscles. 
In addition to protein turnover within individual myofibers, as stated previously, skeletal muscle hypertrophy can also be induced by the activation of muscle satellite cells. In mature muscles, satellite cells are generally quiescent but become activated in response to various stimuli or under muscle regeneration to form new myofibers ${ }^{37}$. When activated, a surge of MRFs, including MyoD, Myf5, and myogenin expression, is required owing to the role of MRFs in driving the differentiation of myoblasts to mature myotubes ${ }^{17}$. Previous studies have shown that mechanical stretching can induce activation of skeletal muscle satellite cells ${ }^{5}$. Elevated expression levels of MRFs have also been observed after shortterm passive repetitive stretching ${ }^{7}$. Pax7 expression has been recognized as a marker of satellite cells. To elucidate whether passive repetitive stretching triggered an active regenerative process that may contribute to senescent skeletal muscle hypertrophy, we first sought to detect Pax $7+$ cells by immunohistochemical analysis. Pax7 + cells were rare, and there was no significant difference in the number of Pax7 + cells between the stretched and unstretched muscles. Therefore, satellite cell content wasn't stimulated by passive repetitive stretching during the experimental period. As also observed in a human study, satellite cell response during post-exercise recovery is blunted with aging ${ }^{38}$. The expression of MyoD was unchanged, whereas the Myf5 and myogenin mRNA expressions were upregulated in the stretched side when compared with the unstretched side. It has also been reported that MRFs mRNA increases occur in muscles, even in the absence of proliferating satellite cells ${ }^{39}$. We are not convinced of the possibility of stretch-induced myogenesis without an increase in MyoD and Pax7 expression levels. Overload-induced muscle hypertrophy requires the involvement of satellite cells in growing mice, whereas it is not necessary for hypertrophic growth in mature adult mice ${ }^{40}$.

\section{Conclusion}

We propose that passive repetitive stretching induces hypertrophy of senescent muscles mainly via regulation of the Akt-mTOR signaling pathway involved in protein turnover. Our study suggests that passive repetitive stretching is an effective measure for sarcopenia prevention and for the maintenance of skeletal muscle mass and function in patients exposed to prolonged inactivity or who are unconscious or paralyzed.

\section{Materials And Methods}

\section{Animals}

We used 35-week-old ${ }^{19}$ male SAM-P8 mice $(n=8)$ for this study. They were housed in plastic cages in a temperature-controlled room with a 12-hour light-dark cycle and provided free access to water and standard food. The experimental procedures were approved by the Animal Care and Use Committees of Hokkaido University. The study protocol was carried out in accordance with the Fundamental Guidelines for Proper Conduct of Animal Experiments and Related Activities in Academic Research Institutions, under the jurisdiction of the Ministry of Education, Culture, Sports, Science and Technology, Japan. A 


\section{Stretching protocol}

The mice were anesthetized with isoflurane solution (FUJIFILM Wako Pure Chemical Corporation, Japan) using inhalation anesthesia equipment (NARCOBIT-E-II KN-1071, Natsume Seisakusho, Japan). The right gastrocnemius muscles were stretched by manual ankle dorsiflexion with knee extended position and within the natural range of motion to avoid muscle damage. Stretching was performed repeatedly, 15 times/min for 15 min daily, 5 days a week for 2 weeks (Figure 1). Contralateral unstretched muscles were examined as controls.

\section{Tissue collection and histology}

Twenty-four hours after the final stretch session, the gastrocnemius muscles of both legs of the mice were removed under deep anesthesia. After weighing, the gastrocnemius muscles were divided into two blocks. Samples for RNA extraction were immediately preserved in liquid nitrogen and stored at $-80^{\circ} \mathrm{C}$. Samples intended for histology were collected in Tissue-Tek ${ }^{\circledR}$ O.C.T. compound (Sakura Finetek, Japan) for frozen sections.

SAM-P8 mice gastrocnemius muscle transversal cross-section $(8 \mu \mathrm{m})$ frozen sections were cut using Cryostat HM550 (Thermo Fisher Scientific, USA) and stained with hematoxylin and eosin (H\&E) to determine the muscle fiber size and frequency distribution. Tissue slides were observed using PALM MicroBeam IV (ZEISS, Germany). The cross-sectional area of the myofibers was calculated from 200 myofibers per muscle sample using the ImageJ software.

\section{Immunohistochemistry}

Muscle fiber type and Pax7-positive nuclei were determined using the ImmunoCruz ${ }^{\circledR}$ rabbit $A B C$ Staining System (sc-2018; Santa Cruz Biotechnology, USA) according to the manufacturer's guidelines. Briefly, 8 $\mu \mathrm{m}$ frozen muscle sections were preincubated for $5 \mathrm{~min}$ in $\mathrm{H}_{2} \mathrm{O}_{2}$ and washed twice for 5 min in PBS and blocked for $1 \mathrm{~h}$ at room temperature. Subsequently, the sections were incubated overnight at $4^{\circ} \mathrm{C}$ with primary antibodies for type 1 muscle fiber (PAD418Mu02, Cloud-Clone Corp, USA, 1:500), type 2A muscle fiber (PAA755Mu01, Cloud-Clone Corp, USA, 1:500), type 2B muscle fiber (PAD416Mu01, Cloud-Clone Corp, USA, 1:500), and Pax7 (AP10488B, Abcepta, USA, 1:100). On the next day, sections were washed three times for $5 \mathrm{~min}$ in PBS and incubated with biotinylated secondary antibody for $1.5 \mathrm{~h}$. After washing in PBS, the sections were incubated for 30 min with $A B$ enzyme reagent. Sections were then washed and incubated in three drops of peroxidase substrate for $5 \mathrm{~min}$. Finally, sections were washed in deionized $\mathrm{H}_{2} \mathrm{O}$ for 5 min, dipped in 90/95/99.5\% ethanol and xylene, and mounted with coverslips using mounting reagent (NEW M·X, Tokyo Garasu Kikai, Japan). Tissue slides were observed using PALM MicroBeam IV (ZEISS, Germany). The fiber-type distribution and number of Pax7-positive nuclei per muscle fiber was calculated as a percentage (200 fibers) using the ImageJ software.

\section{RNA Isolation and Real-time Polymerase Chain Reaction}


A hand homogenizer with Trizol Reagent (Thermo Fisher Scientific) was used to homogenize the muscle tissues, followed by the addition of 0.2 -fold chloroform, a precipitator to extract total RNA from the supernatant, and the removal of protein and deoxyribonucleic acid.

One-step real-time reverse transcription polymerase chain reaction was performed using the Power SYBR Green RNA-to-CT ${ }^{\mathrm{TM}}$ 1-Step Kit (Applied Biosystems, USA) with the following steps: $48^{\circ} \mathrm{C}$ for $30 \mathrm{~min}, 95^{\circ} \mathrm{C}$ for $10 \mathrm{~min}$, followed by 40 cycles at $95^{\circ} \mathrm{C}$ for $15 \mathrm{~s}$, with an annealing and extension step at $60^{\circ} \mathrm{C}$ for 1 min. The primers used are shown in Table 1. GAPDH was used as a housekeeping gene.

\section{Statistics}

The Wilcoxon signed-rank test was used to assess significance. A p-value of $<0.05$ was considered significant. Statistical analysis was performed using GraphPad Prism (version 8) and SPSS Statistics (version 26). Data are presented as mean \pm standard error of the mean (SEM).

\section{Data Availability}

The datasets generated during and/or analysed during the current study are available from the corresponding author on reasonable request.

\section{Declarations}

\section{Author contributions}

Y. W. performed the experiments, analyzed data as well as wrote the manuscript. S. I. designed the research, performed the experiments, analyzed data and contributed with writing of the manuscript. K. I. supervised the study. All authors reviewed the manuscript.

\section{Funding}

This work was supported by JSPS KAKENHI Grant Number JP16K01445 .

\section{Additional Information}

Competing Interests: The authors declare no competing interests.

\section{References}

1. Cruz-Jentoft, A. J. et al. Sarcopenia: European consensus on definition and diagnosis: Report of the European Working Group on Sarcopenia in Older People. Age and Ageing. 39, 412-423(2010).

2. Cruz-Jentoft, A. J. \& Sayer, A. A. Sarcopenia. Lancet. 393, 2636-46 (2019).

3. Williams, P. E. Use of intermittent stretch in the prevention of serial sarcomere loss in immobilized muscle. Rheum. Dis. 49, 316-317 (1990). 
4. Marshall, P. W., Cashman, A. \& Cheema, B. S. A randomized controlled trial for the effect of passive stretching on measures of hamstring extensibility, passive stiffness, strength, and stretch tolerance. Sci. Med. Sport.14, 535-40 (2011).

5. Tatsumi, R., Sheehan, S. M., Iwasaki, H., Hattori, A. \& Allen, R. E. Mechanical Stretch Induces Activation of Skeletal Muscle Satellite Cells in Vitro. Cell. Res. 267, 107-14 (2001).

6. Ikeda, S., Yoshida, A., Matayoshi, S. \& Tanaka, N. Repetitive stretch induces c-fos and myogenin mRNA within several hours in skeletal muscle removed from rats. Phys. Med. Rehabil. 84, 419-23 (2003).

7. Kamikawa, Y., Ikeda, S., Harada, K., Ohwatashi, A. \& Yoshida, A. Passive repetitive Stretching for a short duration within a week increases myogenic regulatory factors and myosin heavy chain mRNA in rats' skeletal muscles. World J. 2013, 493656 (2013).

8. Mirzoev, T.M., Tyganov, S. A., Petrova, I. O. \& Shenkman, B. S. Acute recovery from disuse atrophy: the role of stretch-activated ion channels in the activation of anabolic signaling in skeletal muscle. Am J. Physiol. Endocrinol. Metab.316, E86-E95 (2019).

9. Agata, N. et al. Repetitive stretch suppresses denervation-induced atrophy of soleus muscle in rats. Muscle Nerve. 39, 456-62 (2009).

10. Sasa, T. et al. Continuous muscle stretch prevents disuse muscle atrophy and deterioration of its oxidative capacity in rat tail-suspension models. Am J. Phys. Med. Rehabil.83, 851-6 (2004)

11. Bajpai, A., Li, R. \& Chen, W. The cellular mechanobiology of aging: from biology to mechanics. $N Y$. Acad. Sci. https://doi.org/10.1111/nyas.14529 (2020)

12. Zotz, T. G. et al. Acute effects of stretching exercise on the soleus muscle of female aged rats. Histochem. 118, 1-9 (2016).

13. Hotta, K. et al. Daily muscle stretching enhances blood flow, endothelial function, capillarity, vascular volume and connectivity in aged skeletal muscle. Physiol. 596, 1903-1917(2018).

14. Mankhong, S. et al. Experimental Models of Sarcopenia: Bridging Molecular Mechanism and Therapeutic Strategy. Cells. 9, 1385 (2020).

15. Schiaffino, S., Dyar, K.A., Ciciliot, S., Blaauw, B. \& Sandri M. Mechanisms regulating skeletal muscle growth and atrophy. J. 280, 4294-314 (2013).

16. Rodriguez, J. et al. Myostatin and the skeletal muscle atrophy and hypertrophy signaling pathways. Life. Sci. 71, 4361-71 (2014).

17. Perry, R. L. \& Rudnick, M. A. Molecular mechanisms regulating myogenic determination and differentiation. Biosci. 5, D750-67 (2000).

18. Sousa-Victor, P. \& Muñoz-Cánoves, P. Regenerative decline of stem cells in sarcopenia. Aspects. Med. 50, 109-17 (2016).

19. Guo, A. Y. et al. Muscle mass, structural and functional investigations of senescence-accelerated mouse P8 (SAMP8). Anim. 64, 425-33 (2015). 
20. Vandenburgh, H. \& Kaufman, S. In vitro model for stretch-induced hypertrophy of skeletal muscle. Science. 203, 265-8 (1979).

21. Antonio, J. \& Gonyea, W. J. Progressive stretch overload of skeletal muscle results in hypertrophy before hyperplasia. Appl. Physiol (1985). 75, 1263-71 (1993).

22. Russo, T. L. et al. Stretching and electrical stimulation reduce the accumulation of MyoD, myostatin and atrogin-1 in denervated rat skeletal muscle. Muscle. Res. Cell. Motil. 31, 45-57 (2010).

23. Peviani, M., Gomes, A. R., Moreira, R. F., Moriscot, A. S. \& Salvini, T. F. Short bouts of stretching increase Myo-D, myostatin and atrogin-1 in rat soleus muscle. Muscle Nerve. 35, 363-70 (2007).

24. Baewer, D. V. et al. Passive stretch inhibits central corelike lesion formation in the soleus muscles of hindlimb-suspended unloaded rats. Appl. Physiol (1985). 97, 930-4 (2004).

25. Talbot, J. \& Maves, L. Skeletal muscle fiber type: using insights from muscle developmental biology to dissect targets for susceptibility and resistance to muscle disease. Interdiscip. Rev. Dev. Biol. 5, 518-34 (2016).

26. Hornberger, T. A. et al. Mechanical stimuli regulate rapamycin-sensitive signalling by a phosphoinositide 3-kinase-, protein kinase B-and growth factor-independent mechanism. Biochem J.380, 795-804 (2004).

27. Bodine, S. C. et al. Akt/mTOR pathway is a crucial regulator of skeletal muscle hypertrophy and can prevent muscle atrophy in vivo. Cell. Biol. 3, 1014-9 (2001).

28. Sakamoto, K., Aschenbach, W. G., Hirshman, M. F. \& Goodyear, L. J. Akt signaling in skeletal muscle: regulation by exercise and passive stretch. J. Physiol. Endocrinol. Metab. 285, E1081-8 (2003).

29. Baar, K., Torgan, C. E., Kraus, W. E. \& Esser, K. Autocrine phosphorylation of p70(S6k) in response to acute stretch in myotubes. Cell. Biol. Res. Commun. 4, 76-80 (2000).

30. Thomson, D. M. \& Gordon, S. E. Impaired overload-induced muscle growth is associated with diminished translational signaling in aged rat fast-twitch skeletal muscle. Physiol. 574, 291-305 (2006).

31. Soares, A. G. et al. Ubiquitin-ligase and deubiquitinating gene expression in stretched rat skeletal muscle. Muscle Nerve. 36, 685-93 (2007).

32. Altun, M. et al. Muscle wasting in aged, sarcopenic rats is associated with enhanced activity of the ubiquitin proteasome pathway. Biol. Chem. 285, 39597-608 (2010).

33. Clavel, S. et al. Atrophy-related ubiquitin ligases, atrogin-1 and MuRF1 are up-regulated in aged rat Tibialis Anterior muscle. Ageing. Dev. 127, 794-801 (2006).

34. Whitman, S. A., Wacker, M. J., Richmond, S. R. \& Godard, M. P. Contributions of the ubiquitinproteasome pathway and apoptosis to human skeletal muscle wasting with age. Arch. 450, 437-46 (2005).

35. Edström, E., Altun, M., Hägglund, M. \& Ulfhake, B. Atrogin-1/MAFbx and MuRF1 are downregulated in aging-related loss of skeletal muscle. J. Gerontol. A. Biol. Sci. Med. Sci. 61, 663-74 (2006). 
36. Aoki, M. S., Soares, A. G., Miyabara, E. H., Baptista, I. L. \& Moriscot, A. S. Expression of genes related to myostatin signaling during rat skeletal muscle longitudinal growth. Muscle Nerve. 40, 992-9 (2009).

37. Bazgir, B., Fathi, R., Rezazadeh Valojerdi, M., Mozdziak, P. \& Asgari, A. Satellite cells contribution to exercise mediated muscle hypertrophy and repair. Cell J. 18, 473-484 (2017).

38. Snijders, T. et al. The skeletal muscle satellite cell response to a single bout of resistance-type exercise is delayed with aging in men. Age (Dordr). 36, 9699 (2014).

39. Lowe, D. A. \& Always, S. E. Stretch-induced myogenin, MyoD, and MRF4 expression and acute hypertrophy in quail slow-tonic muscle are not dependent upon satellite cell proliferation. Tissue. Res.296, 531-9 (1999).

40. Murach, K. A. et al. Differential requirement for satellite cells during overload-induced muscle hypertrophy in growing versus mature mice. Skelet Muscle. 7, 14 (2017).

\section{Tables}

Table 1 Sequences of real-time PCR primers used

\begin{tabular}{|c|c|c|}
\hline Genes & Forward primer & Reverse primer \\
\hline MyoD & 5'-CCCCGGCGGCAGAATGGCTACG-3' & 5'-GGTCTGGGTTCCCTGTTCTGTGT-3' \\
\hline Myf5 & 5'-GAGGGAACAGGTGGAGAACTATTA-3' & 5'-CGCTGGTCGCTGGAGAG-3' \\
\hline myogenin & 5'-ACTCCCTTACGTCCATCGTG-3' & 5'-CAGGACAGCCCCACTTAAAA-3' \\
\hline Myostatin & 5'-CTGTAACCTTCCCAGGACCA-3' & 5'-TCTTTTGGGTGCGATAATCC-3' \\
\hline Akt & 5'-GCCCTCAAGTACTCATTCCAG-3' & 5'-ACACAATCTCCGCACCATAG-3' \\
\hline p70s6K & 5'-TGAGTCAAGCCTTGGTCGAG-3' & 5'-AAGAGTCGAGAGAGACGCCC-3' \\
\hline 4E-BP1 & 5'-CGGAAGATAAGCGGGCAG-3' & 5'-CAGTGTCTGCCTGGTATGAG-3' \\
\hline MAFbx & 5'-CTCTGCTGTGAGTGCCACAT-3' & 5'-CAATGAGCCTGGGTACCACT-3' \\
\hline MuRF1 & 5'-TGGAAACGCTATGGAGAACC-3' & 5'-AACGACCTCCAGACATGGAC-3' \\
\hline GAPDH & 5'-TGACGTGCCGCCTGGAGAAA-3 & 5'-AGTGTAGCCCAAGATGCCCTTCAG-3 \\
\hline
\end{tabular}

\section{Figures}




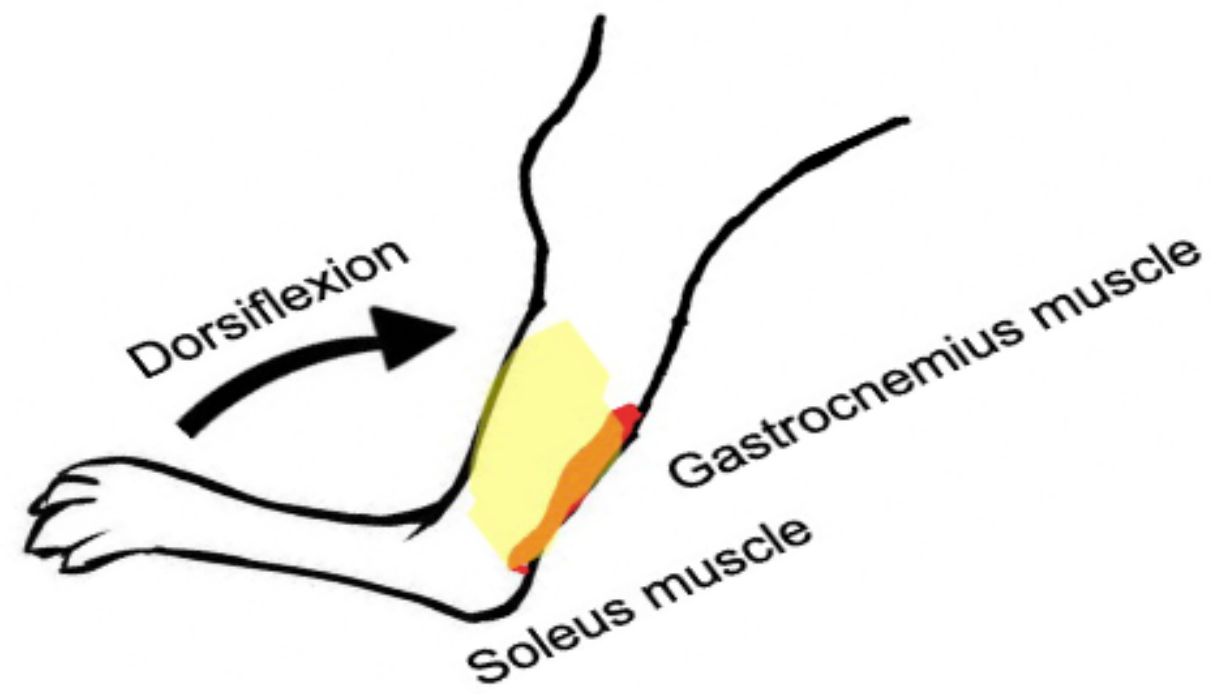

Figure 1

Muscle passive repetitive stretching by ankle dorsiflexion under knee extended position 
A

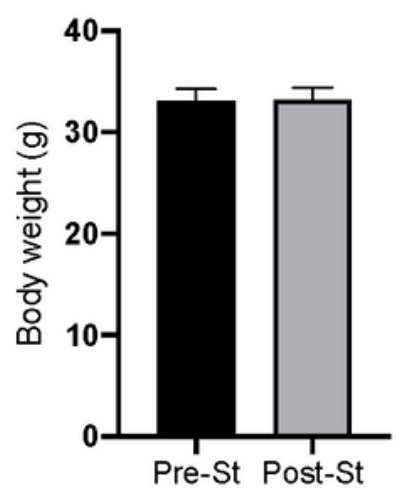

C
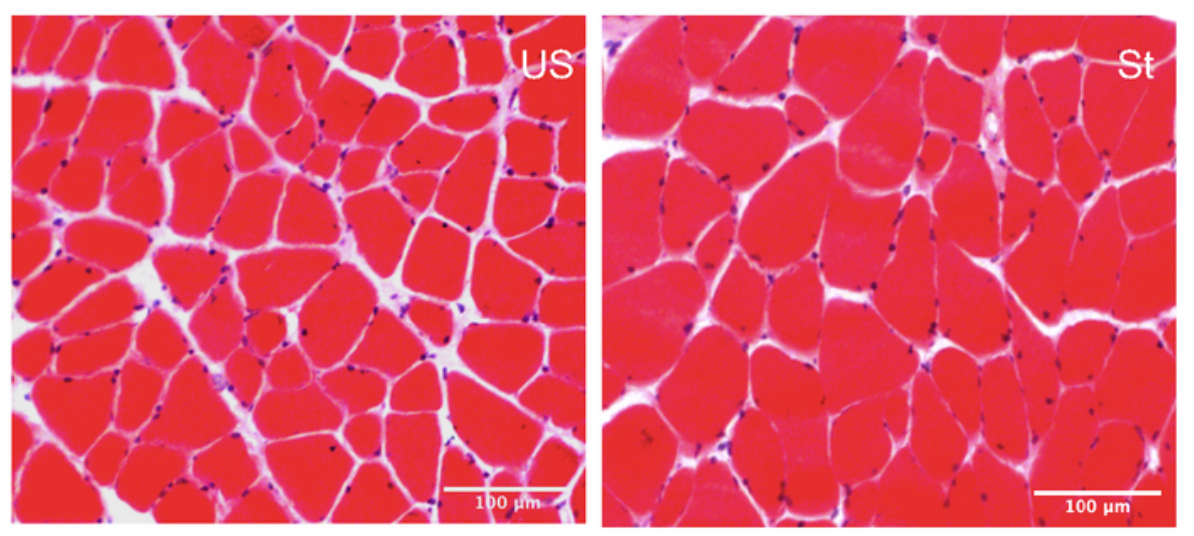

D

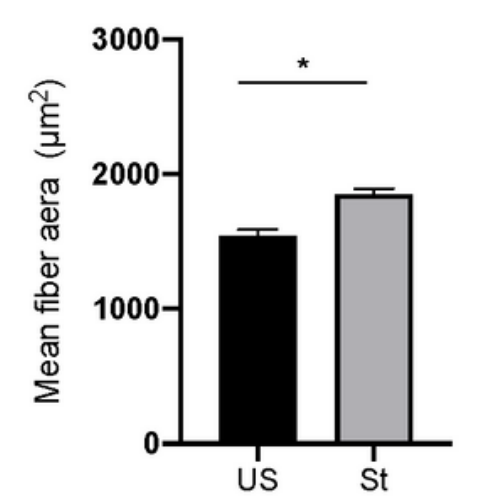

B

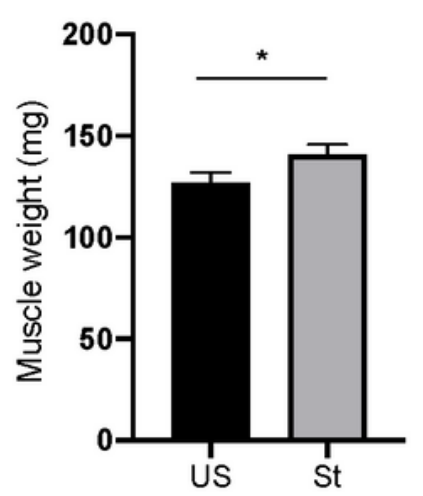

E

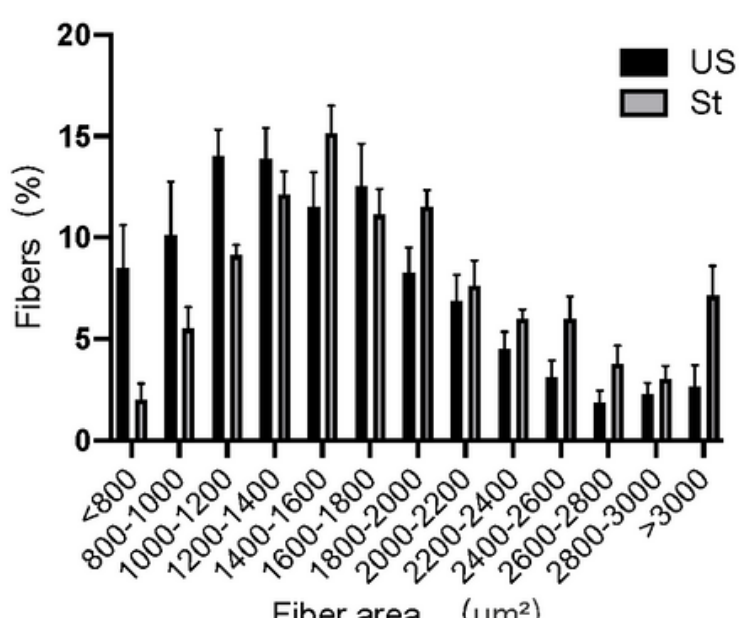

Fiber area $\left(\mu \mathrm{m}^{2}\right)$

\section{Figure 2}

Body weight, muscle weight and fiber analysis (A) Body weight. (B) Mean weight of gastrocnemius muscle. (C) H\&E staining of the gastrocnemius muscle. The bar indicates $100 \mu \mathrm{m}$. Magnification: 100x.

(D) Mean cross-sectional fiber area of gastrocnemius muscle. (E) Frequency distribution of crosssectional gastrocnemius muscle fiber area. US: unstretched. St: stretched. Values are expressed as mean \pm SEM. ${ }^{*} p<0.05, n=8 /$ group. 
A
Type 2A
Type 2B
Type 1
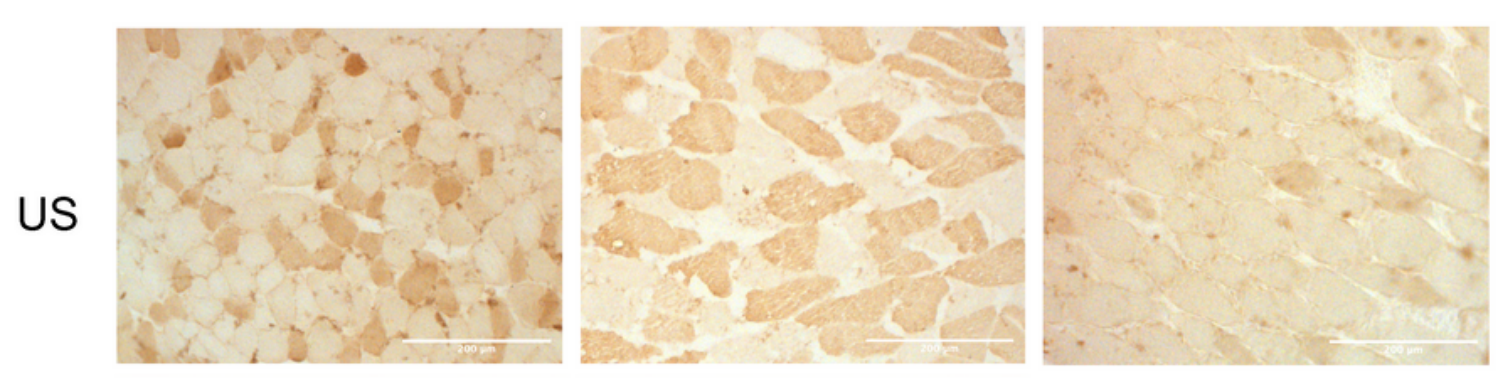

St
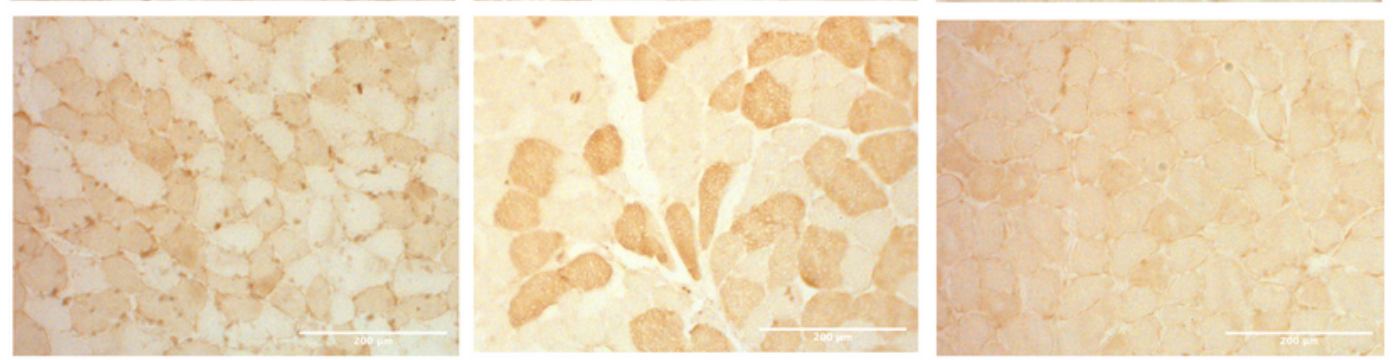

B

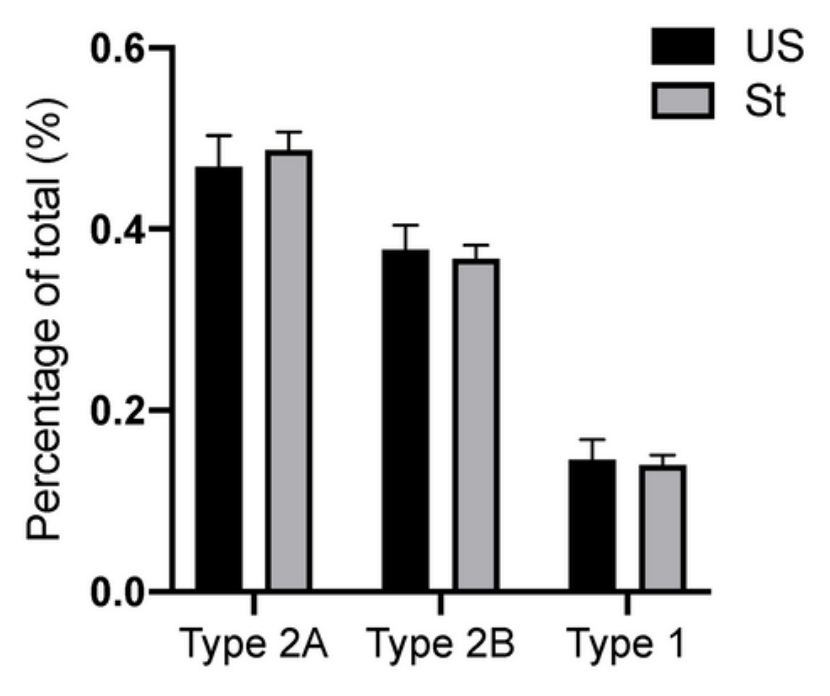

Figure 3

Immunohistochemical analysis of muscle fiber type (A) Representative images are shown for muscle fiber type 2A, type 2B, type 1, visualized with DAB (brown). The bar indicates $200 \mu \mathrm{m}$. Magnification: 100x. (B) Distribution of fiber types in gastrocnemius muscle. US: unstretched. St: stretched. Values are expressed as mean \pm SEM. ${ }^{\star} p<0.05, n=8$ /group. 
A
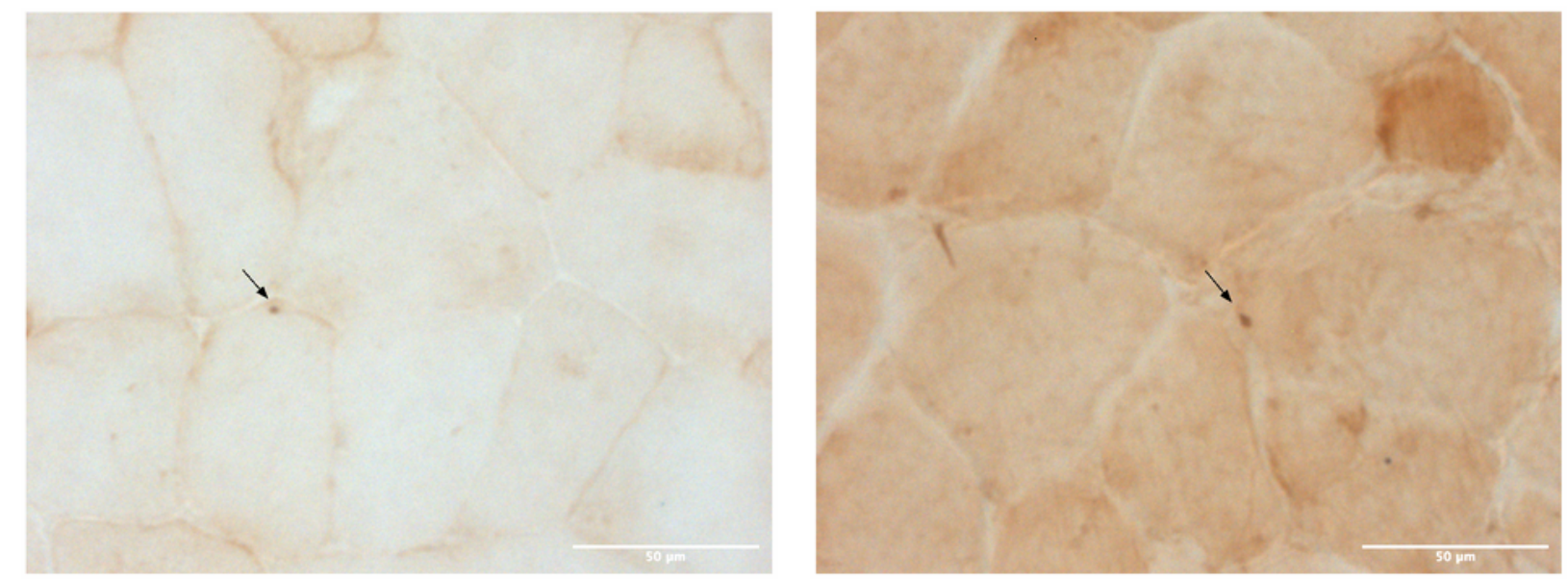

B

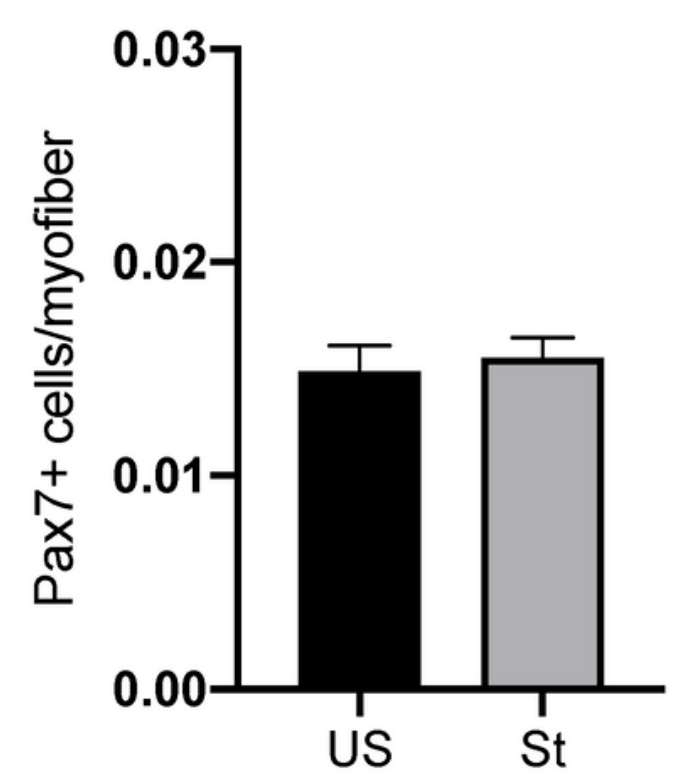

Figure 4

Immunohistochemical analysis of Pax7+ cells (A) Representative images showing Pax7+ myonuclei (Arrowheads). The bar indicates $50 \mu \mathrm{m}$. Magnification: 400x. (B) Quantification of the number of satellite cells (Pax7+ myonuclei/fiber) in gastrocnemius muscle. US: unstretched. St: stretched. Values are expressed as mean \pm SEM. ${ }^{*} p<0.05, n=8 /$ group. 
A

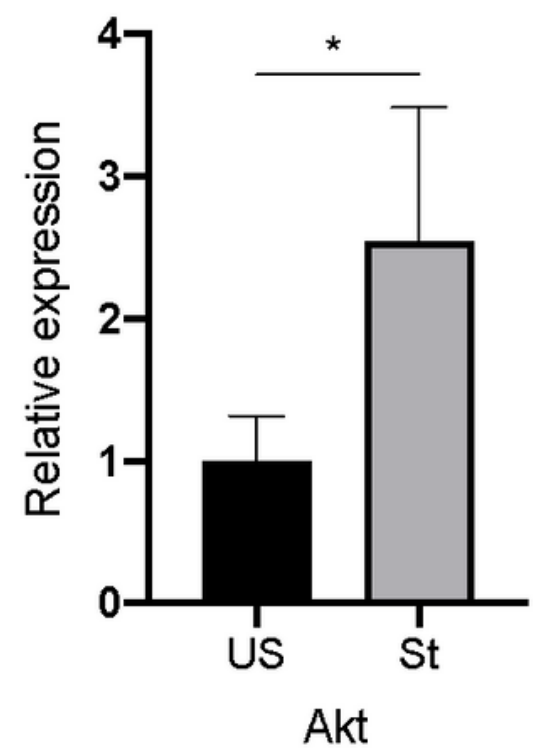

D

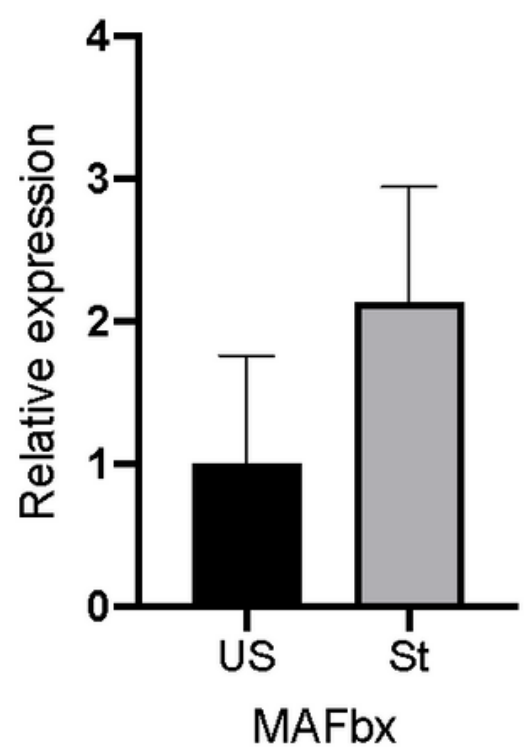

B

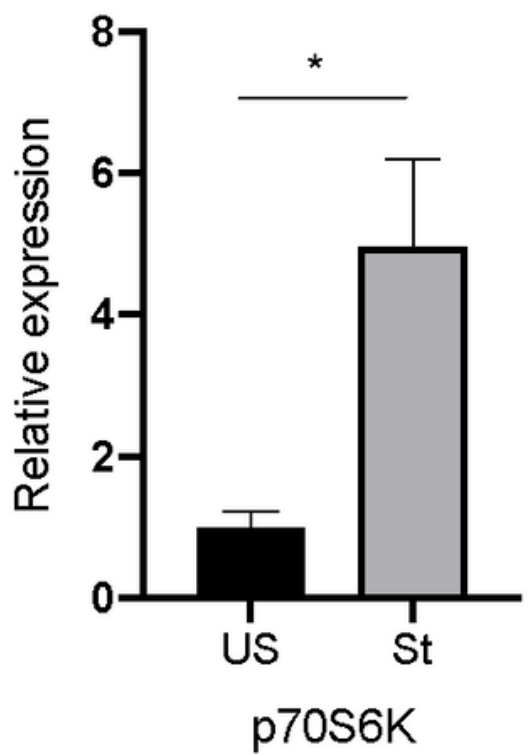

E

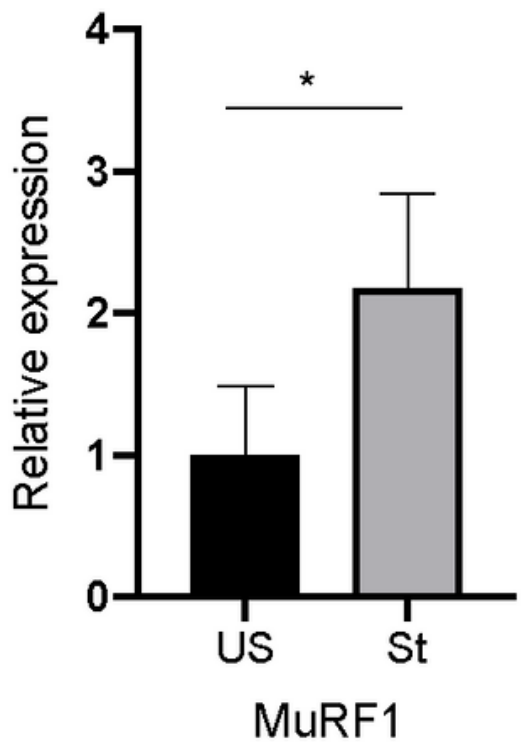

Figure 5

Real-time PCR gene expression of Akt, p70S6K, 4E-BP1, and muscle-specific E3 ubiquitin ligases MAFbx and MuRF1. US: unstretched. St: stretched. Values are expressed as mean \pm SEM. ${ }^{*} p<0.05, n=8 /$ group. 
A

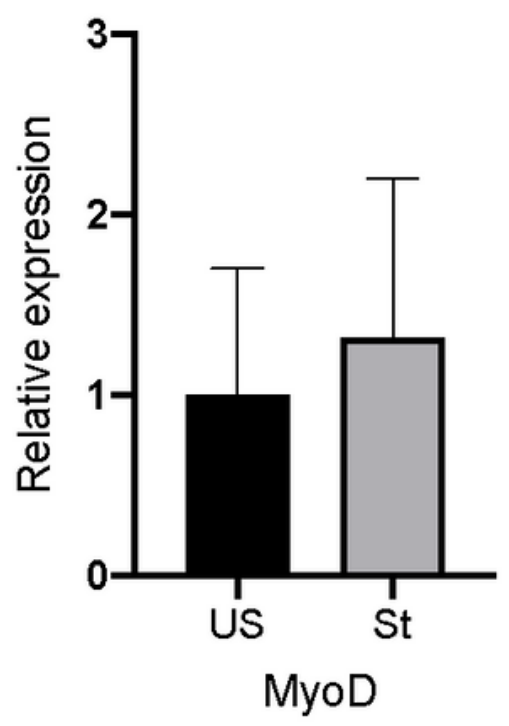

C

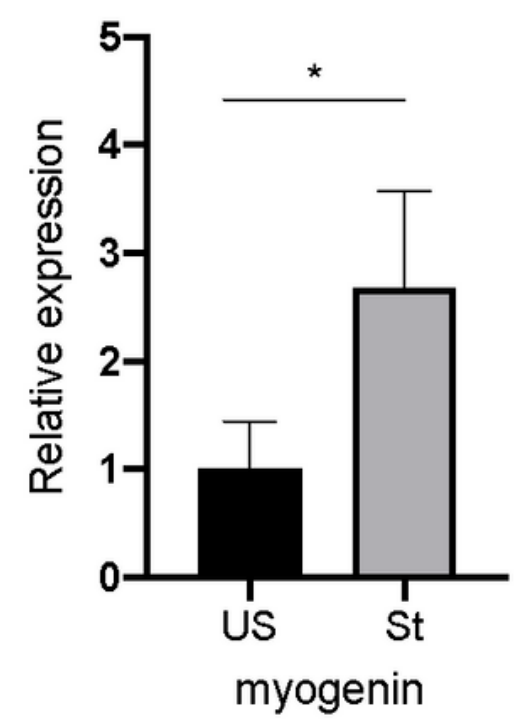

B

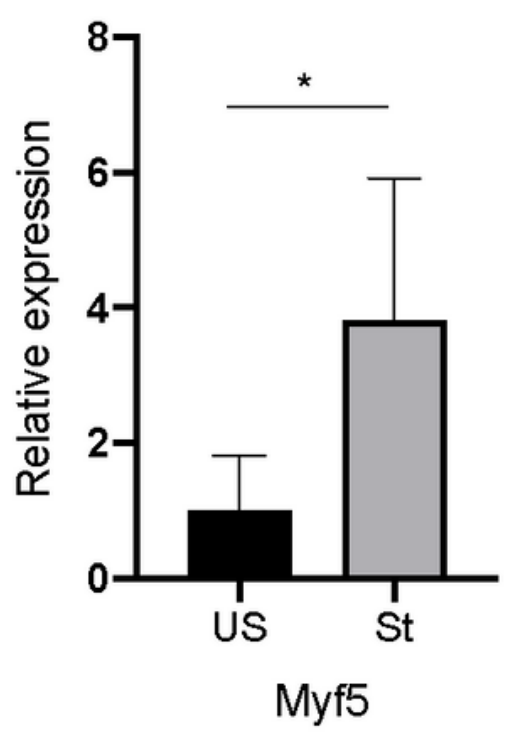

D

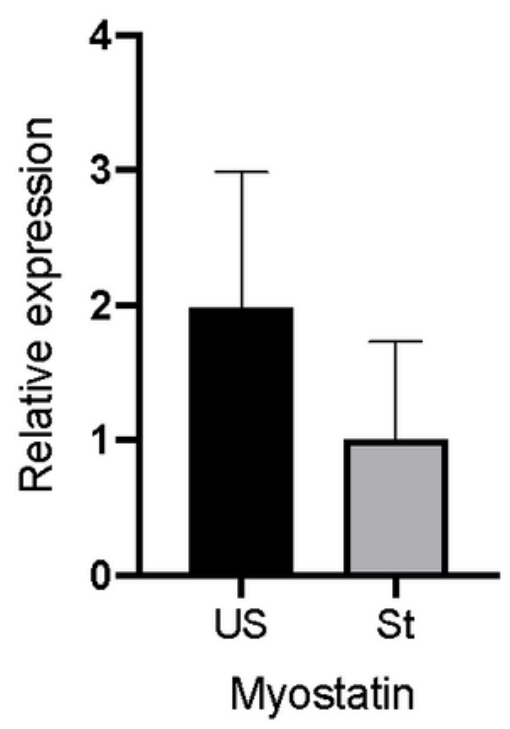

Figure 6

Real-time PCR gene expression of Myogenic regulatory factors and Myostatin. US: unstretched. St: stretched. Values are expressed as mean \pm SEM. ${ }^{*} p<0.05, n=8 /$ group. 